Technical Note

\title{
The influence of contact line velocity and acceleration on the dynamic contact angle: An experimental study in microgravity
}

\author{
Sheng-Hua Xu, Cai-Xia Wang, Zhi-Wei Sun*, Wen-Rui Hu \\ Key Laboratory of Microgravity, Institute of Mechanics, Chinese Academy of Sciences, Beijing 100190, PR China \\ National Microgravity Laboratory, Institute of Mechanics, Chinese Academy of Sciences, Beijing 100190, PR China
}

\section{A R T I C L E I N F O}

\section{Article history:}

Received 21 June 2010

Received in revised form 15 January 2011

Accepted 17 January 2011

\section{Keywords}

Microgravity

Contact line

Dynamic contact angle

Interior angle

\begin{abstract}
A B S T R A C T
In this study, a novel experimental method by using the short-time microgravity condition created by the drop tower facility is proposed to investigate the dependence of dynamic contact angle on both the velocity and acceleration of contact line. The values of the dynamic contact angle with respect to different velocity and acceleration of contact line were determined under some different parameters. Our experimental results show that the dynamic contact angle depends not only on the velocity of the contact line but also its acceleration.
\end{abstract}

(c) 2011 Elsevier Ltd. All rights reserved.

\section{Introduction}

The problems on contact line's motion and dynamic contact angle have received significant interest and attention due to many natural and industrial processes involving the flow of fluid over the solid, such as a raindrop hitting a windowpane and wetting films $[1,2]$. The influence of dynamic contact angle on the related dynamic wetting, such as the case of a droplet impacting a solid surface, have been extensively studied up to date $[3,4]$. And the dynamic contact angle is a determinant factor for the forces on interface and the behavior of fluid motion [5] in microgravity.

The value of dynamic contact angle is commonly thought to be related with the Capillary number $\mathrm{Ca}(=\mu v / \sigma)$ and the static contact angle $[6,7]$. Therefore, the contact line velocity, viscosity and surface tension [6-8] are all important parameters that influence the dynamic contact angle. For the influence of moving contact line, only velocity $v$ was taken into account in most studies [6,7]. However, there possibly exists acceleration of the moving contact line for the fluid flow. Theoretical studies of contact angle in unsteady flow have implied that the acceleration is also a parameter correlative to the dynamic contact angle [9]. Unfortunately, due to

\footnotetext{
* Corresponding author at: Key Laboratory of Microgravity, Institute of Mechanics, Chinese Academy of Sciences, Beijing 100190, PR China. Tel.: +86 1082544093 ; fax: +8610 82544096

E-mail address: sunzw@imech.ac.cn (Z.-W. Sun).
}

the lack of more experimental studies on the dependence of dynamic contact angle on the acceleration of the contact line, the experimental formulas without acceleration [7] were still applied in discussions of flow relevant to dynamic contact angle no matter whether the acceleration exists or not $[5,10]$. Therefore, it is worthwhile to specially investigate whether and how both the velocity and acceleration of the moving contact line affect the dynamic contact angle.

In the present paper, we first propose a novel experimental method for measuring the dynamic contact angle under microgravity condition using drop tower. Utilizing this method, the dynamic contact angle with respect to different velocity and acceleration is studied experimentally.

\section{Description of the measurement}

Upon entering microgravity status, capillary forces govern the flow and the reorientation of the free surface towards the new equilibrium position often occurs, going with the contact line motion. In this study, we will use such capillary flow in microgravity to study the dynamic contact angle in interior corners, which is more universal than circular ones that can be thought of as with interior angle $180^{\circ}$. According to the theory by Concus and Finn [11], only when static contact angle $\theta_{s}>90^{\circ}-\alpha$ can an equilibrium configuration exist in the interior angle $2 \alpha$ (as shown in Fig. 1) under microgravity condition. Therefore, the parameters in this study are chosen to satisfy this condition (which will be described in next section) to utilize the interface movement from the equilibrium in 


\section{Nomenclature}

a contact line acceleration, $\mathrm{mm} \mathrm{s}^{-2}$

$d \quad$ the face widths of the test tubes, $\mathrm{cm}$

$\mathrm{Ca}$ capillary number

$h \quad$ tip location, $\mathrm{mm}$

$h_{0} \quad$ the initial liquid height in tubes, $\mathrm{mm}$

$t \quad$ microgravity time, $\mathrm{s}$

$v \quad$ contact line velocity, $\mathrm{mm} \mathrm{s}^{-1}$

$V_{0} \quad$ initial liquid volume in quadrate container, $\mathrm{m}^{3}$

Greek symbols dynamic viscosity, $\mathrm{N} \mathrm{s} \mathrm{m}^{-2}$

surface tension, $\mathrm{N} \mathrm{m}^{-1}$

half of interior angle of the container, ${ }^{\circ}$

contact angle, ${ }^{\circ}$

equilibrium (static) contact angle, ${ }^{\circ}$

dynamic contact angle, ${ }^{\circ}$

the angle of the contact line with the edges of the containers, ${ }^{\circ}$

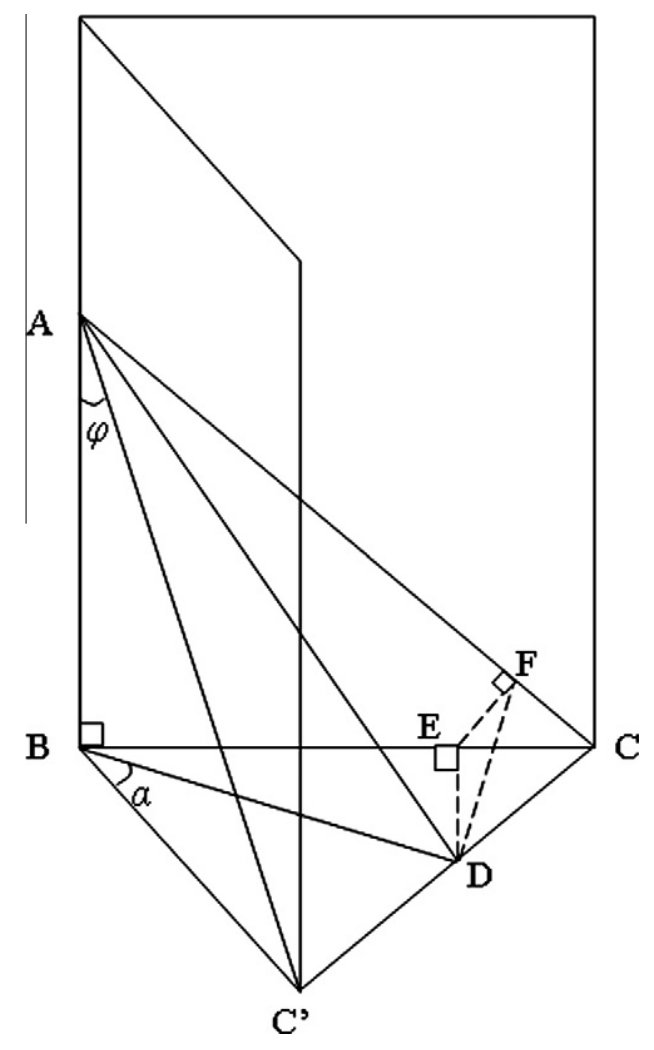

Fig. 1. Schematic drawing of geometrical lines in an interior corner.

normal gravity to that in microgravity to study the dynamic contact angle.

Fig. 1 schematically shows the condition that the corner is partially filled by fluid with contact angle $\theta$. In this figure, the two sides $A B C$ and $A B C^{\prime}$ form an interior angle $C B C^{\prime}=2 \alpha$, and $\mathrm{CBD}=\mathrm{C}^{\prime} \mathrm{BD}=\alpha$. The lines $\mathrm{AC}$ and $\mathrm{AC}^{\prime}$ are the tangent lines of the contact lines on the two sides, so that the angles $B A C=\mathrm{BAC}^{\prime}=\varphi$, which can be measured directly from experiments.

By point $\mathrm{D}$ we draw a straight line $\mathrm{DE}$, perpendicular to $\mathrm{BC}$ and intercept $\mathrm{BC}$ at $\mathrm{E}$. It can be seen that $\mathrm{DE}$ is perpendicular to the surface $A B C$, so that $D E$ is also perpendicular to line $A C$. By point $E$ we draw a straight line EF, perpendicular to $A C$ and intercept $A C$ at F. Since $A C$ is perpendicular to $E F$ and $D E$, it is perpendicular to the surface DEF so that DF is also perpendicular to AC. Therefore, the value of the angle DFE will be equal to the contact angle $\theta$.
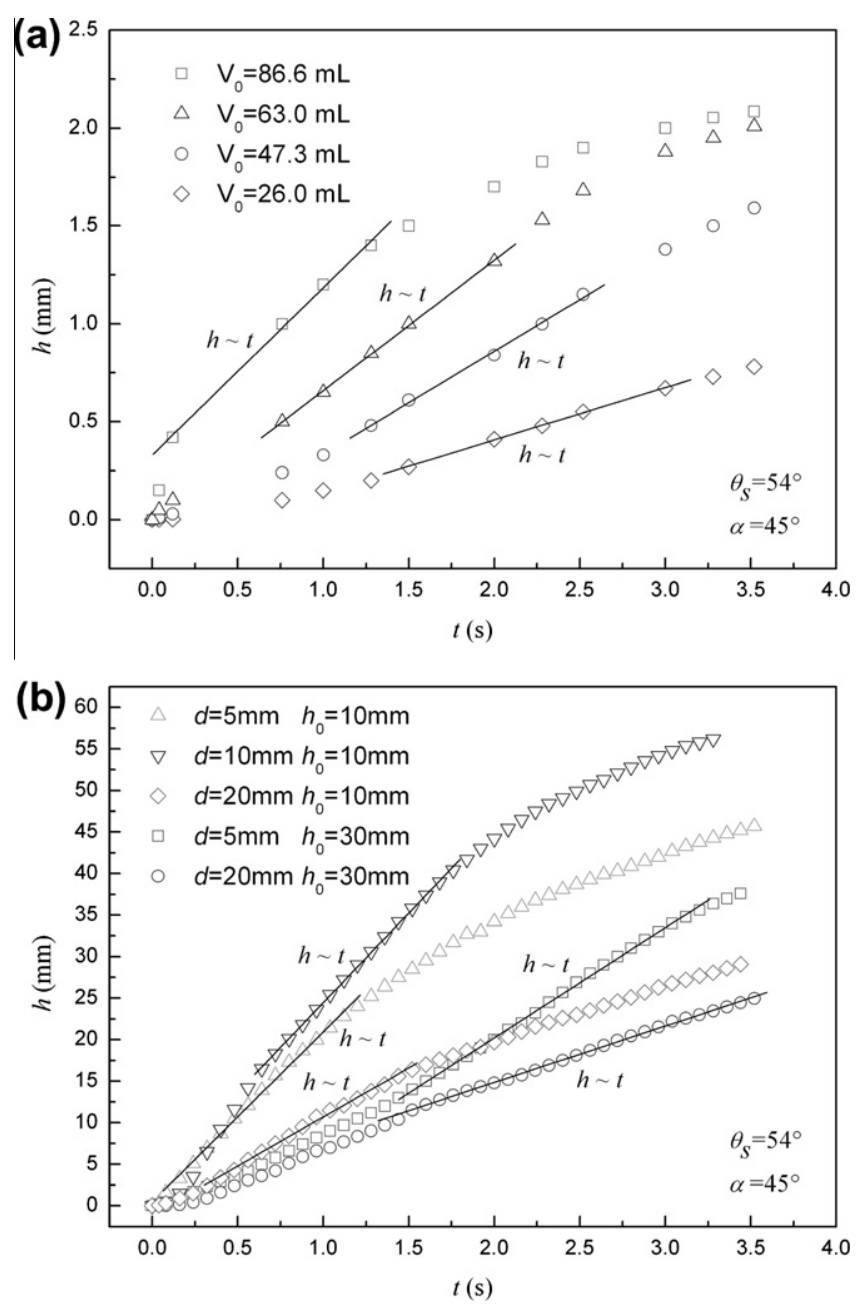

Fig. 2. The tip location (height) $h(\mathrm{~mm})$ as a function of microgravity time $t(\mathrm{~s})$ : (a) for different initial liquid volume $\left(V_{0}\right)$ in quadrate container, (b) for different tube size $(d)$ and initial liquid height $\left(h_{0}\right)$ in quadrate tubes. Test liquid: Eth/ $\mathrm{H}_{2} \mathrm{O}$ 30/70 (\%vol.). The lines show the duration when the flow is in constant velocity.

From Fig. 1, we can get $\mathrm{FE}=\mathrm{CE} * \cos (\varphi)$ and $\mathrm{DE}=\mathrm{CE} * \operatorname{ctan}(\alpha)$, so that $\tan (\mathrm{DFE})=\mathrm{DE} / \mathrm{FE}=\operatorname{ctan}(\alpha) / \cos (\varphi)=\sec (\varphi) / \tan (\alpha)$. Therefore, by geometry analysis, the relationship of the contact angle can be determined by:

$\tan (\theta)=\sec (\varphi) / \tan (\alpha)$

where $\theta, \varphi$ and $\alpha$ are the contact angle, the measured angle of the contact line with edge and half angle of the interior corner, respec- 
tively. Using this formula, the dynamic contact angle at the interior corner can be evaluated from $\alpha$ and measured $\varphi$.

\section{Experimental}

The experiments were conducted in Beijing drop tower. This facility provides $3.6 \mathrm{~s}$ of free fall with $g$-residual estimated at $10^{-5} g_{0}$. The test liquid is aqueous ethanol mixture: Eth $/ \mathrm{H}_{2} \mathrm{O}$ 30/70 (\%vol.). The density, surface tension and kinematic viscosity of the test liquid are $937 \mathrm{~kg} \mathrm{~m}^{-3}, 0.0364 \mathrm{~N} \mathrm{~m}^{-1}$ and $1.83 \mathrm{cSt}$, respectively [12]. The equilibrium contact angle $\theta_{\mathrm{s}}$ for Eth/ $\mathrm{H}_{2} \mathrm{O}$ $30 / 70 \%$ on PMMA is $54^{\circ}$, which was measured by Optical Contact Angle Measuring Device (OCA20) with the SCA-software. In experiments, the capillary flow processed in two kinds of systems: quadrate container (container having quadrate cross section with initial liquid volume $V_{0}$ ) and quadrate tube immersed in liquid (the side of quadrate tubes is $d$, and the initial liquid height in tubes is $h_{0}$ ). The cells are all made of solid PMMA [poly(methyl methacrylate)]. The value of $\theta_{\mathrm{s}}=54^{\circ}>45^{\circ}=\left(90^{\circ}-\alpha\right)$ ensures that the condition described in the above section is satisfied. During drop tower experiments, the capillary flow on one side of the container/tube was directly monitored by CCD camera WAT-660D(CCIR) with pixels $537(\mathrm{H}) * 597(\mathrm{~V})$ and recording update rate 25 frame/s and recorded. The detailed information about the experimental setup and the procedure can be seen from our previous studies [12,13], since they are almost the same except for the containers/tubes. From the captured images of one side [12] such as side $A B C^{\prime}$ schematically shown in Fig. 1, $\varphi$ can be measured manually from line $A C^{\prime}$ and edge $\mathrm{AB}$. The dynamic contact angle at different velocity and acceleration can then be evaluated from Eq. (1).

\section{Results and discussion}

From the recorded figures, the tip location $h$ at different time $t$ can be determined, the detail of which can be seen from our previous studies [12,13]. The results of $h$ vs. $t$ are shown in Fig. 2, in which the parameters for quadrate containers and quadrate tubes are given. Fig. 2(a) shows that larger liquid volume $\left(V_{0}\right)$ corresponds to higher velocity in quadrate container, which is in accordance with Ref. [12]. For quadrate tubes, the tube sizes $(d)$ and initial liquid heights $\left(h_{0}\right)$ affect the velocity of tips as shown in

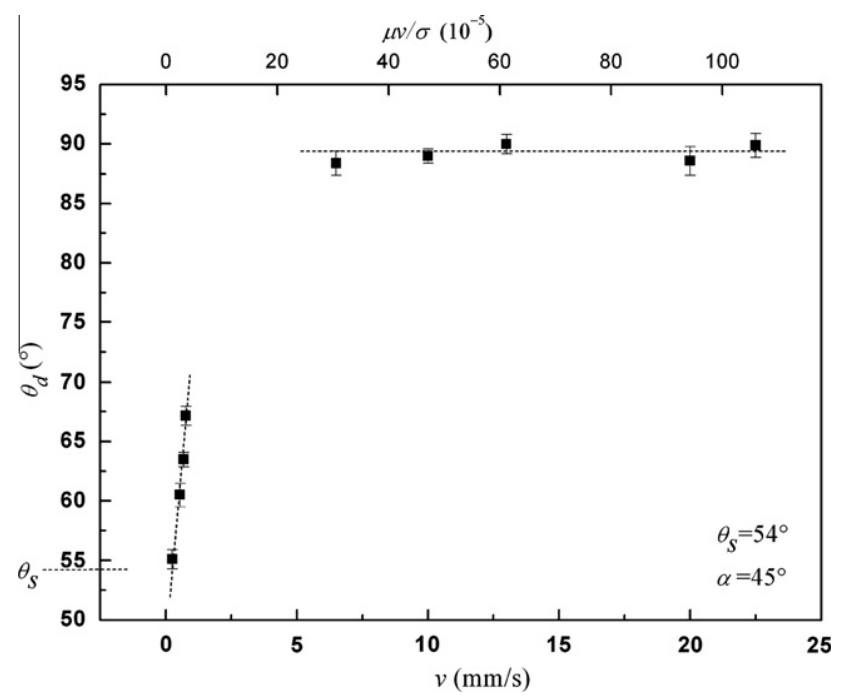

Fig. 3. Experimental results for the dependence of the dynamic contact angle on the contact line velocity without acceleration. The values of the velocities are determined from Fig. 2.
Fig. 2(b), which is compatible with the results for circular tubes [5,13]. Fig. 2(a) and (b) shows that the velocity is constant $(h \sim t)$ during some microgravity time range for the cells used in this study, which is similar to the capillary flow in circular tubes [5]. Therefore, our experiments can study the dynamic contact angle with constant velocities (no acceleration) in microgravity. Since the mechanisms for the capillary flow in container and tubes are different, the velocity ranges for the two kinds of cells are different. For tubes, there is pressure difference between the fluid inside and outside the tube, which results in a higher velocity than the containers.

The velocity dependence of the dynamic contact angle is shown in Fig. 3. The velocity range varies from 0.25 to $0.77 \mathrm{~mm} / \mathrm{s}$ (in quadrate containers with size of $3.5 \mathrm{~cm} \times 4.5 \mathrm{~cm}$ ) and from 6.5 to $22.5 \mathrm{~mm} / \mathrm{s}$ (in quadrate tubes). The corresponding capillary number $\mathrm{Ca}$ varies from $1.2 \times 10^{-5}$ to $3.6 \times 10^{-5}$ and from $31 \times 10^{-5}$ to $106 \times 10^{-5}$. Fig. 3 shows that at low velocities of contact line the dynamic contact angle increases rapidly with the velocity, whereas at high velocities the dynamic contact angle approaches a constant value and presents a plateau over a range of velocities. The changing tendency of the dynamic contact angle with the contact line velocity is consistent with the experimental results on the ground $[3,14]$.

For the time duration when the velocity is not constant, the corresponding velocity and the acceleration of the tip location of the
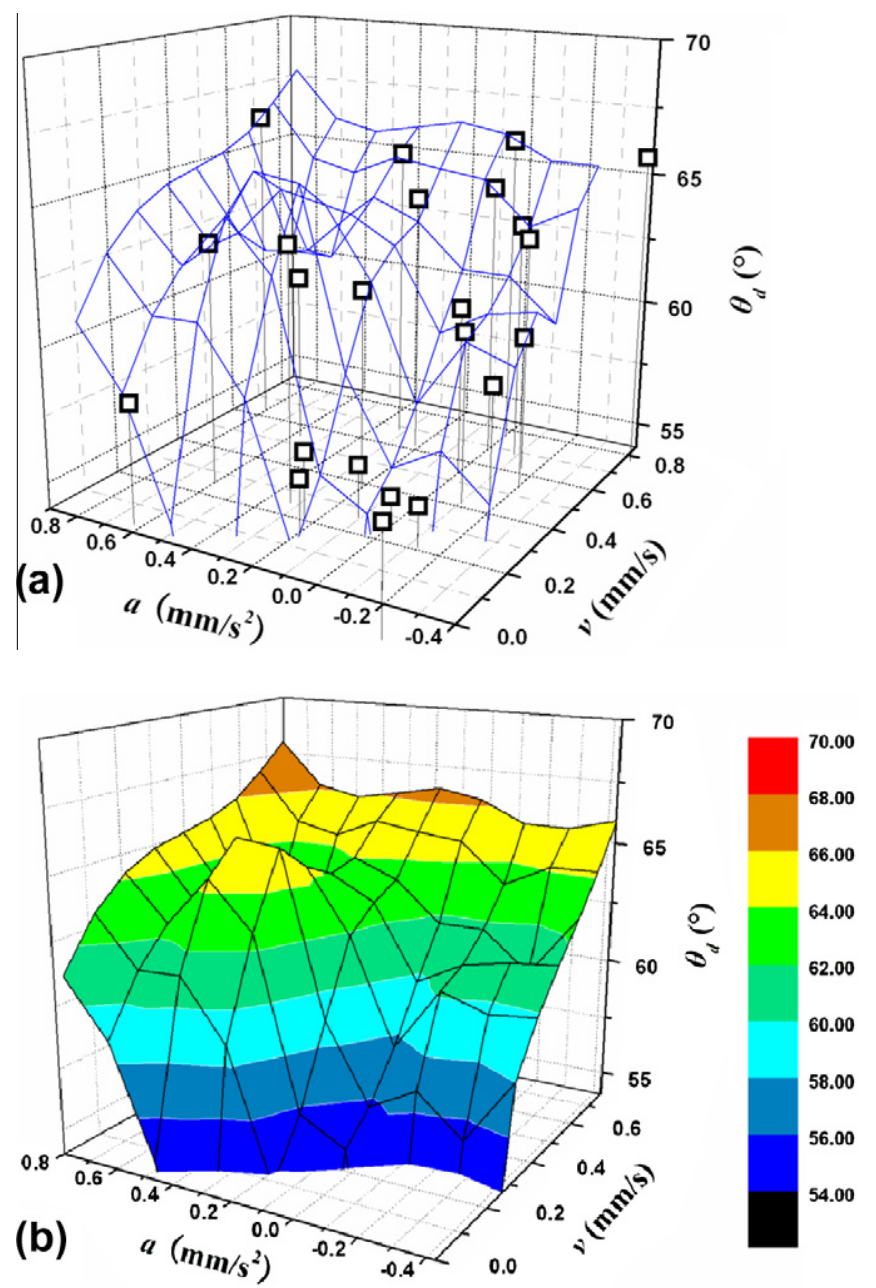

Fig. 4. (a) Typical experimental results for dynamic contact angle as a function of contact line velocity $v$ and acceleration $a$ using quadrate container. (b) 3D color map surface. 

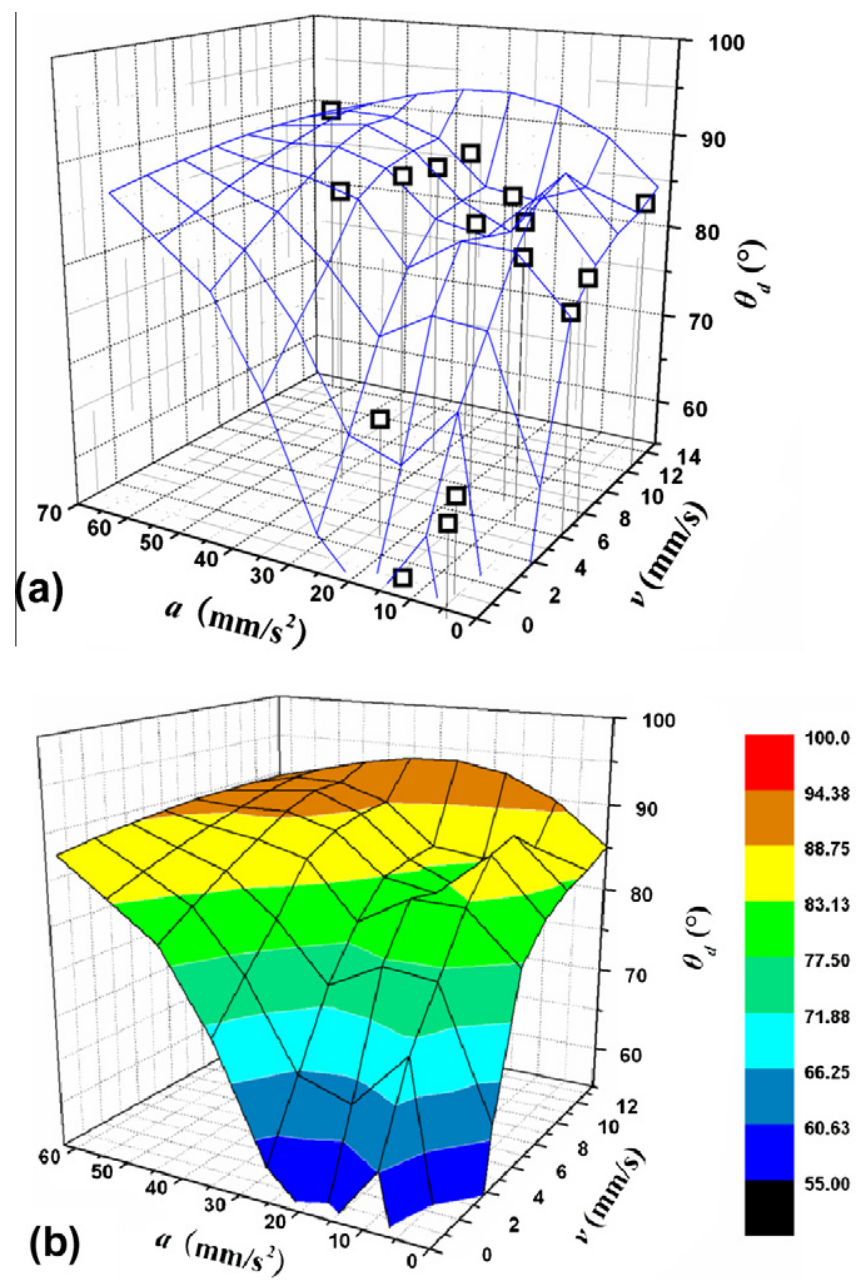

Fig. 5. (a) Typical experimental results for dynamic contact angle as a function of contact line velocity $v$ and acceleration $a$ using quadrate tubes with $d=20 \mathrm{~mm}$. (b) 3D color map surface.

meniscus in corner can be achieved from $\Delta h / \Delta t$ and $\Delta(\Delta h / \Delta t) / \Delta t$. By measuring $\varphi$, the dynamic contact angle can be calculated according to Eq. (1). So the dynamic contact angles for different velocities and accelerations can be acquired, as shown in Figs. 4 and 5. In Fig. 4 the velocity ranges from 0.025 to $0.77 \mathrm{~mm} / \mathrm{s}$ and the acceleration ranges from -0.425 to $0.8 \mathrm{~mm} / \mathrm{s}^{2}$, while in Fig. 5 the velocity ranges from 0.5 to $13.7 \mathrm{~mm} / \mathrm{s}$ and the acceleration ranges from 0 to $62.5 \mathrm{~mm} / \mathrm{s}^{2}$. Figs. 4(a) and 5(a) correspond to the data of contact angle using quadrate container and quadrate tubes, respectively. And the corresponding 3D color map surfaces obtained using a Renka-Cline interpolation are shown in Figs. 4(b) and 5(b), respectively. These figures display a changing tendency that the dynamic contact angle increases with the increase of the velocity (see through the axis of velocity) and acceleration (see through the axis of acceleration) of the contact line.

\section{Conclusion}

In this study, we present a new approach to study the dependence of dynamic contact angle on contact line movement in microgravity by utilizing the liquid flow in interior corner. In this method, the velocity and acceleration of the contact lines can be easily adjusted by changing corresponding parameters and therefore it is particularly suitable for short duration microgravity experiments. By means of this new experimental approach, we measured the dynamic contact angle with respect to different contact line velocities and accelerations under microgravity condition. The experimental results presented in this paper show that not only the contact line velocity but also its acceleration have effect on the dynamic contact angle. Especially, the dynamic contact angle is larger for larger acceleration of the contact line.

\section{Acknowledgments}

This work is supported by grants (Numbers 11032011 and 10972217) from the National Natural Science Foundation of China and the Knowledge Innovation Program of the Chinese Academy of Sciences (including Grant No. KJCX2-YW-L08).

\section{References}

[1] I.S. Bayer, C.M. Megaridis, Contact angle dynamics in droplets impacting on flat surfaces with different wetting characteristics, J. Fluid Mech. 558 (2006) 415.

[2] E. Rio, A. Daerr, B. Andreotti, L. Limat, Boundary conditions in the vicinity of a dynamic contact line: experimental investigation of viscous drops sliding down an inclined plane, Phys. Rev. Lett. 94 (2005) 024503.

[3] K. Yokoi, D. Vadillo, J. Hinch, L. Hutchings, Numerical studies of the influence of the dynamic contact angle on a droplet impacting on a dry surface, Phys. Fluids 21 (2009) 072102.

[4] D.C. Vadillo, A. Soucemarianadin, C. Delattre, D.C.D. Roux, Dynamic contact angle effects onto the maximum drop impact spreading on solid surfaces, Phys. Fluids 21 (2009) 122002.

[5] M. Stange, M.E. Dreyer, H.J. Rath, Capillary driven flow in circular cylindrical tubes, Phys. Fluids 15 (2003) 2587.

[6] R.L. Hoffman, A study of the advancing interface. Part I: Interface shape in liquid-gas systems, J. Colloid Interface Sci. 50 (1975) 288.

[7] T.S. Jiang, S.G. Oh, J.C. Slattery, Correlation for dynamic contact angle, J. Colloid Interface Sci. 69 (1979) 74.

[8] T.S. Meiron, A. Marmur, I.S. Saguy, Contact angle measurement on rough surfaces, J. Colloid Interface Sci. 274 (2004) 637.

[9] Oleg V. Voinov, Meniscus contact angle in unsteady viscous flow, J. Colloid Interface Sci. 201 (1998) 93.

[10] N. Fries, M. Dreyer, The transition from inertial to viscous flow in capillary rise, J. Colloid Interface Sci. 327 (2008) 125.

[11] P. Concus, R. Finn, On capillary free surfaces in the absence of gravity, Acta Math. 132 (1974) 177.

[12] C.X. Wang, S.H. Xu, Z.W. Sun, W.R. Hu, A study of the influence of initial liquid volume on the capillary flow in interior corner under microgravity, Int. J. Heat Mass Trans. 53 (2010) 1801.

[13] C.X. Wang, S.H. Xu, Z.W. Sun, W.R. Hu, Influence of contact angle and tube size on capillary-driven flow under microgravity, AIAA J. 47 (2009) 2642.

[14] A.M. Schwartz, S.B. Tejada, Studies of dynamic contact angles on solid, J. Colloid Interface Sci. 38 (1972) 359. 